\title{
FTIR Analysis of Biodegradation of Polystyrene by Intestinal Bacteria Isolated from Zophobas Morio and Tenebrio Molitor
}

\author{
Hong-Han Lin, Hsiao-Han Liu* \\ Department of Biological Science \& Technology, I-Shou University, Kaohsiung, Taiwan \\ Received 02 April 2020; received in revised form 03 September 2020; accepted 06 November 2020 \\ DOI: https://doi.org/10.46604/peti.2021.5450
}

\begin{abstract}
Since the 1970s, polystyrene (PS) plastic marine pollution has become a global concern. Tenebrio molitor and Zophobas morio beetle larva actively respond to a diet of primarily polystyrene. The tantalizing evidence indicates that the gut bacteria of these beetle larva enables them not only to consume polystyrene, but to successfully biodegrade polystyrene. Heretofore, data collection to verify polystyrene degradation by the gut bacteria of these larva has taken up to six months per test. Our laboratory created a platform by dissolving PS into a liquid mineral culture medium to examine PS degradation by the gut bacteria. Under investigation, PS works as main carbon source supporting the growth of gut bacteria. Fourier-transform Infrared spectroscopy (FTIR) is an effective method which can provide relevant information on chemical changes. This study presents a methodology using FTIR and Visible Light Spectrometry as an efficient methodology to verify the physiological degradation of polystyrene.
\end{abstract}

Keywords: polystyrene, tenebrio molitor, zophobas morio, FTIR

\section{Introduction}

Polystyrene (PS) marine pollution had intensively increased since the 1970s [1]. Plastic contributes to 60-90\% of marine debris and $50 \%$ of debris belongs to the single-use plastics (SUPs), including polystyrene [2]. Recent publications suggest that weathering processes promote further fragmentation of PS into nano-plastics, and show a metabolic toxicity which can change the behavior of marine creatures, or damage human health [3-4].

Previous research successfully isolated two bacterial strains from the intestines of Tenebrio molitor and Zophobas morio larval worms. Turbidity assay evidence suggested that these bacteria have the ability to digest polystyrene and successfully survive [5]. With two potentially PS-degrading enzymes identified in the foregoing research, the efficacy and enzymatic catalyzing mechanism were still under investigation. That is the research that this paper takes up.

Our laboratory adopted the naming conventions of "ZM1"for the bacterial strains from Zophobas morio and "TM1" for the strains taken from Tenebrio molitor. 16sR As sequence of each revealed the taxonomic status of TM1 to be genus Pantoea and that of ZM1 to be genus Klebsiella. ZM1 has a 99\% genetic similarity to Klebsiella pneumoniae DSM 30104; TM1 has a 96\% genetic similarity to Pantoea agglomerans JCM 1236 [5].

With these potentially PS-degrading strains identified, they were cultured in a medium with polystyrene (PS) as the sole carbon source, making the TM1 and ZM1 no option for sustenance other than Polystyrene. According to our data, TM1 and ZM1 began to synthesize bacterial enzymes that seem capable to successfully degrading the PS-medium [5]. Fourier-transform

* Corresponding author. E-mail address: hansliu@isu.edu.tw 
Infrared Spectroscopy (FTIR) has been commonly applied in the field of biochemistry as a powerful technique to measure structural changes of organic and inorganic molecules and their functional groups [6-10].

In order to digest polystyrene as a carbon source, a secretion of the specific enzymes that can bind a polystyrene substrate which is required for the survival of the intestinal bacteria. Therefore, if any enzymatic catalyzation of the carbon bonds in the PS-medium occurred, FTIR could be a potential tool for measuring these molecular changes and demonstrating the sites where enzymes tend to activate [6].

\section{Materials and Methods}

\subsection{Isolating polystyrene-degrading microbes}

The experiments began by acclimating the Tenebrio molitor and Zophobas morio larval worms to a polystyrene diet for a period of 21 days (Fig. 1). PS-degrading bacterial strains TM1 and ZM1 were then isolated from the intestines by selective PS agar plates [5].

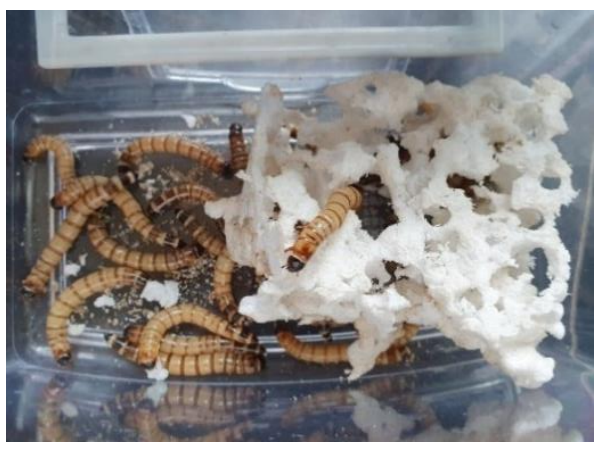

(a) Zophobas morio

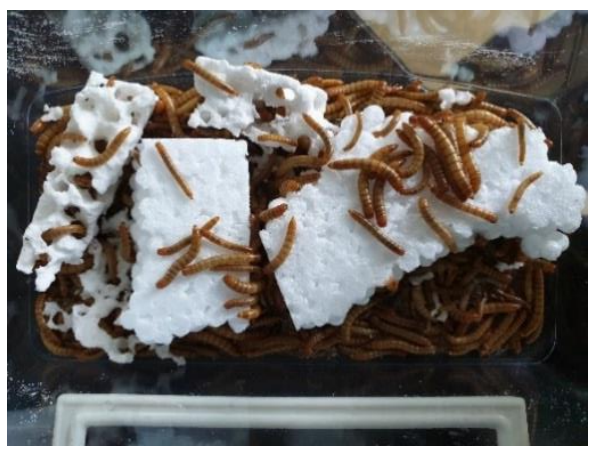

(b) Tenebrio molitor

Fig. 1 Two larval worms' species subsisting on Polystyrene

\subsection{Bacterial cultivation and PS-biodegradation experiments}

The intestinal bacterial strains ZM1 and TM1 were inoculated and incubated within the PS-medium. A customized platform for polystyrene biodegradation testing of TM1 and ZM1 from the previously cited research was used [5]. The PS-medium was $50 \mathrm{ml}$, with $45 \mathrm{ml}$ of inorganic salt basal buffer, $5 \mathrm{ml}$ of polystyrene emulsion, and $0.05 \mathrm{ml}$ of $38 \%$ yeast extract. Chloroform is used to dissolve polystyrene into emulsion, but after that it will be vaporized, so the final product doesn't contain chloroform.

At this stage, three tubes of PS-medium were loaded in $50 \mathrm{ml}$ centrifuge tubes; two of them were inoculated with bacterial strains ZM1 and TM1. Each was cultivated for further FTIR testing. The remaining tube of medium was designated as the un-inoculated control. The FTIR cultivation courses were operated under a stable temperature at $37^{\circ} \mathrm{C}, 144$ hours ( 6 days)

\subsection{Turbidity analysis by absorbance and transparency}

First, quantification and comparison of absorbance and transparency in PS-medium during cultivation course was conducted. Second, the absorbance and transparency levels of the PS-medium were used as a measure of turbidity. Turbidity is considered a quantitative indicator for PS degrading efficiency; analysis of this kind is also referred to as a Turbidity Assay [5, $11]$.

The absorbance of PS-medium was measured by microplate spectrophotometer (Thermo Scientific Multiskan GO), and $200 \mu \mathrm{l}$ of sample were loaded for each measurement: from 230-900 $\mathrm{nm}$. The OD value was measured every $1 \mathrm{~nm}$ of wavelength. Transparency was determined by spectrophotometer (Metertech SP-830), and $1 \mathrm{ml}$ of sample was loaded for each 
test: from 400-700 $\mathrm{nm}$. The percentage of transparency was measured at every $10 \mathrm{~nm}$ of wavelength. All measurements were performed with a time lapse at $0 \mathrm{hr}, 24 \mathrm{hr}$ and $48 \mathrm{hr}$.

\subsection{FTIR analysis}

FTIR spectroscopy was applied to the PS-medium after the cultivation of two strains to determine if enzymatic changes consistent with degradation of carbon bonds took place [12]. To avoid the interference of non-specific organic molecules within the microbes' outcome spectra, the samples (two inoculums and one control) were first centrifuged $\left(4000 \mathrm{rpm}, 25^{\circ} \mathrm{C}, 10\right.$ min) to remove debris and particles.

After centrifugation, $3 \mathrm{ml}$ of supernatant of the medium was dried in a drying oven. The dried sample was then mixed with $\mathrm{KBr}$ salt powder $(0.5 \mathrm{mg}$ of sample plus $20 \mathrm{mg}$ of $\mathrm{KBr})$ and hand-pulverized by mortar; $10 \mathrm{mg}$ of mixture were tableted for FTIR test afterward. FTIR spectroscopy (Perkin-Elmer FT-IR RX1) at 4000-400 $\mathrm{cm}^{-1}$ was performed and the samples were collected at day 0 and day 6 [13].

\section{Result and Discussion}

\subsection{Absorbance and transparency determination}

The turbidity of PS-medium during cultivation was measured based on absorbance and transparency. The absorbance levels of the experimental groups gradually decreased during the cultivation time lapse (Fig. 2), and the transparency rose (Fig. 3), while the control remained stable. This data suggests that the activation of TM1 and ZM1 caused PS to degrade in the medium.

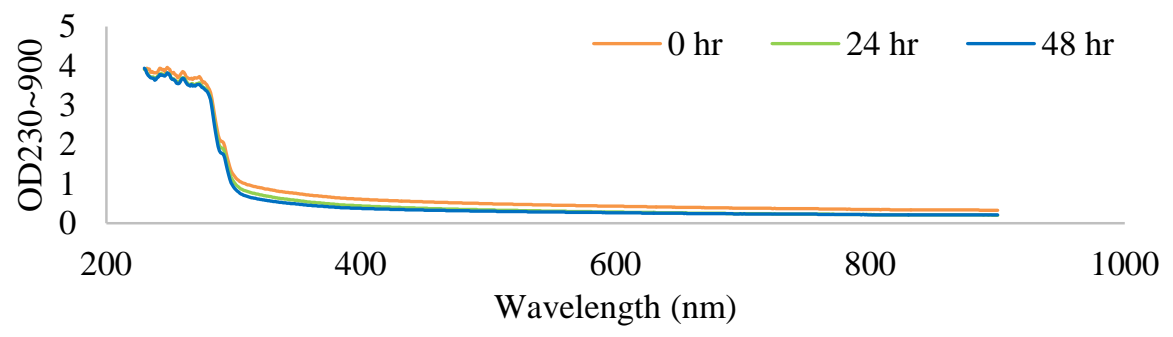

(a) Absorbance with inoculation of strains ZM1

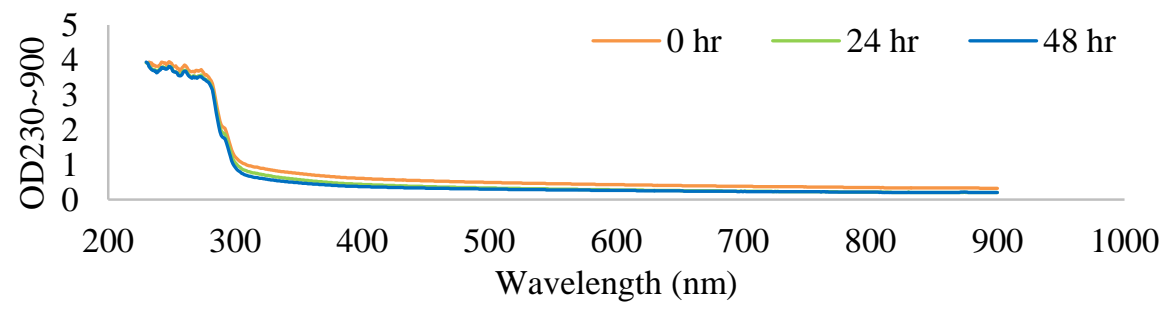

(b) Absorbance with inoculation of strains TM1

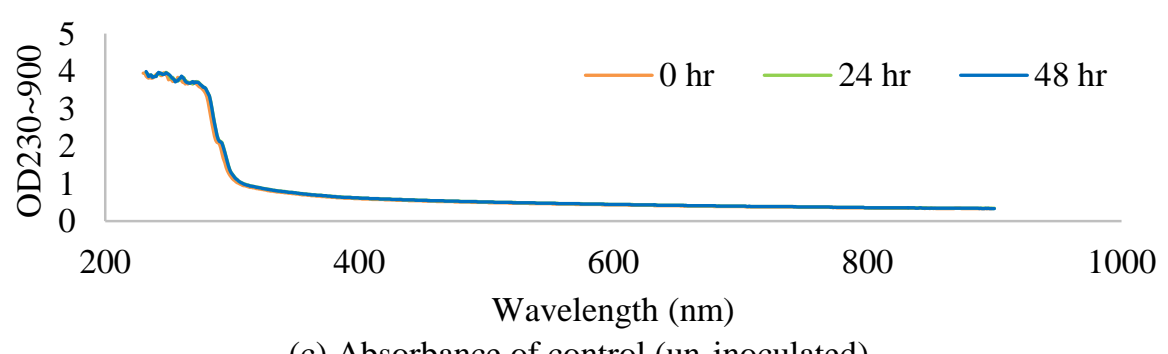

(c) Absorbance of control (un-inoculated)

Fig. 2 Turbidity assay based on absorbance

To demonstrate biological degradation of polystyrene by the gut bacteria of Tenebrio molitor and Zophobas morio beetle larva this study performed turbidity assays based on absorbance and transparency on inoculated TM1 and ZM1 gut bacteria 
strains. The assay data reveal declining turbidity in the PS-medium across the full visible light spectrum, not only at the 600 $\mathrm{nm}$ point as the previous study had described [5].

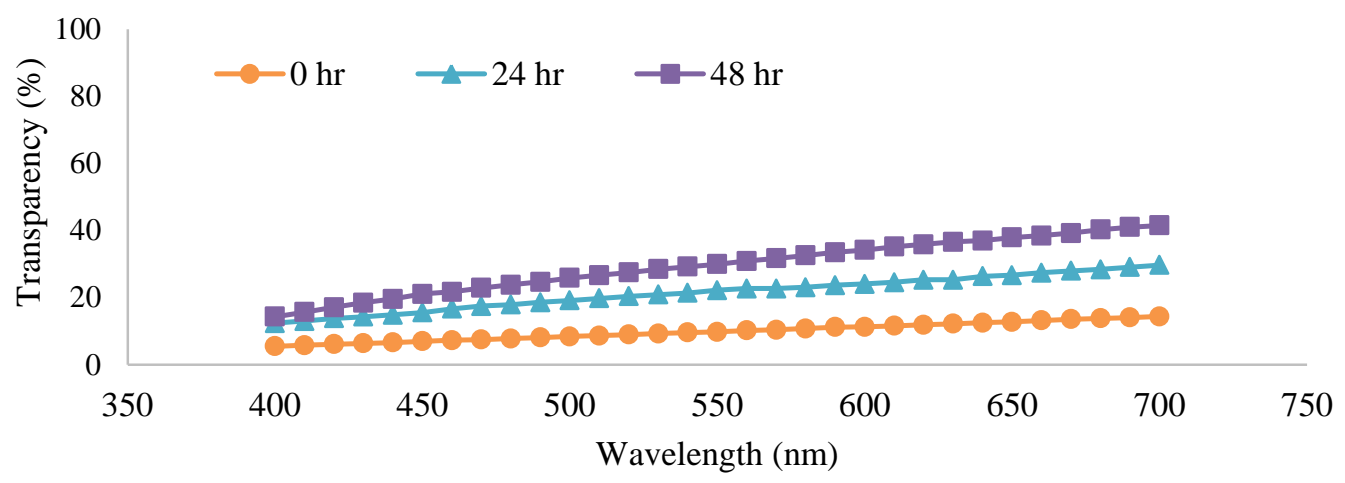

(a) Transparency with inoculation of strains ZM1

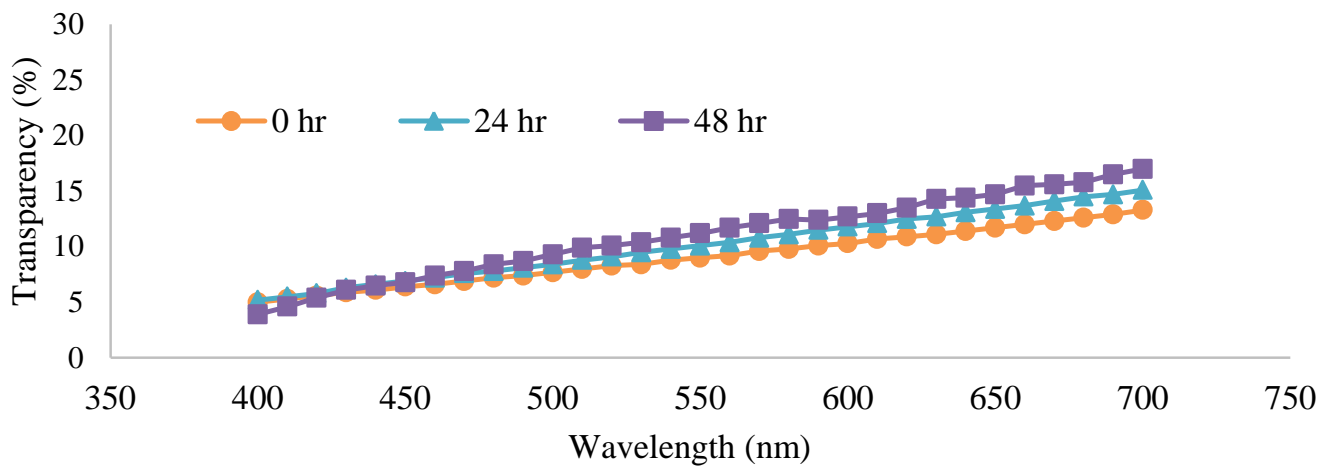

(b) Transparency with inoculation of strains TM1

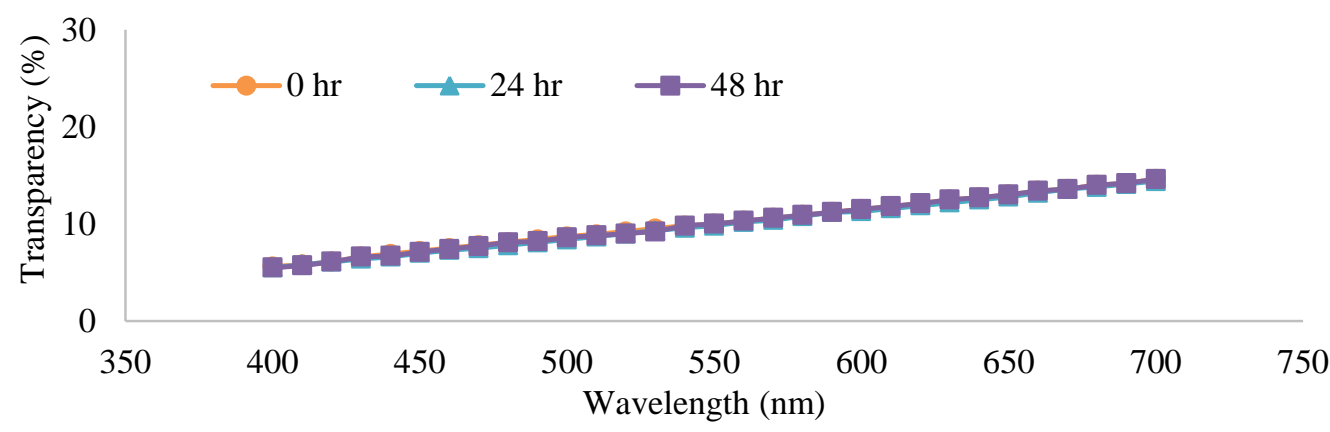

(c) Transparency of control (un-inoculated)

Fig. 3 Turbidity assay based on transparency

$0 \mathrm{hr} 24 \mathrm{hr} 48 \mathrm{hr} 72 \mathrm{hr} 96 \mathrm{hr}$

ZM1

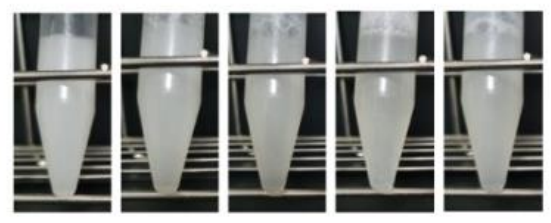

TM1

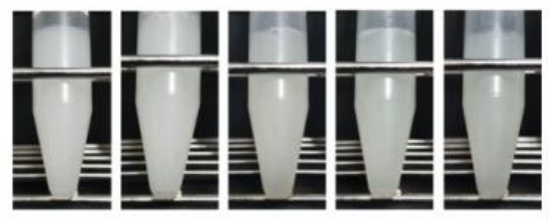

control

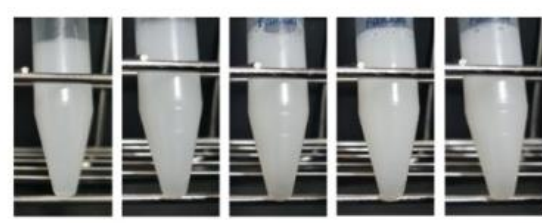

Fig. 4 Visualization of turbidity 


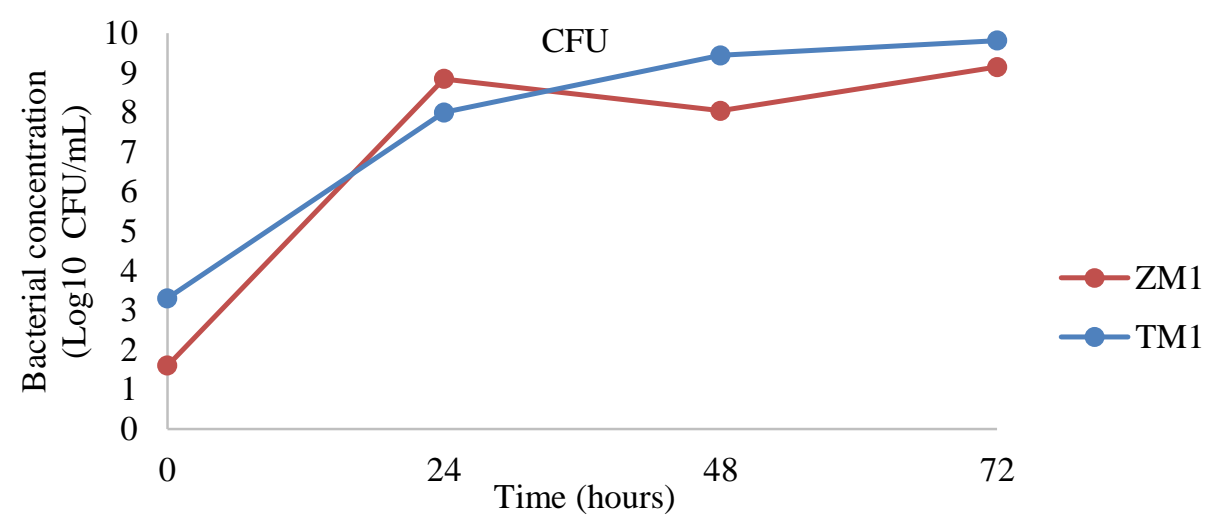

Fig. 5 Bacterial CFU (measured by MacConkey agar plate)

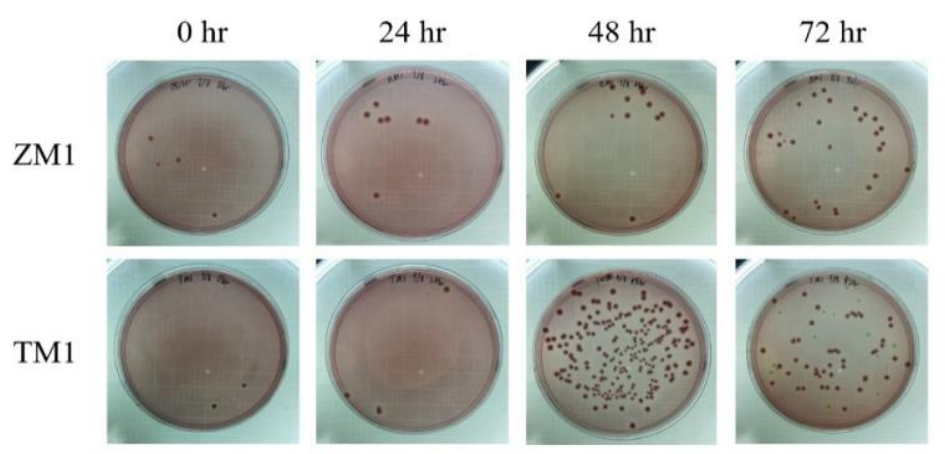

Fig. 6 Bacterial growth curve

A series of photos of strain TM1 and ZM1 growing in PS-medium also shows the changes of turbidity. PS-medium inoculated with TM1 and ZM1 become increasingly transparent from time at $0 \mathrm{hr}$ to $96 \mathrm{hr}$, while the control group remains its originally opaque and turbid (Fig. 4). Figs. 5-6 shows the CFU data (measured by MacConkey agar plate) represent bacterial concentration and growth. In CFU test, the dilution factor of ZM1 at time 0, 24, 48 and 72 hr were $1,10^{7}, 10^{6}$ and $5 \times 10^{6}$, respectively; TM1 at time $0,24,48$ and $72 \mathrm{hr}$ were $10^{2}, 2.5 \times 10^{6}, 10^{6}$ and $10^{7}$, respectively. All colonies were yielded by plating $0.1 \mathrm{ml}$ of diluted sample (Fig. 5).

\subsection{FTIR analysis}

Fig. 7 shows an FTIR spectrum of the PS medium which is inoculated with TM1 and ZM1. Fig. 7(c) shows the un-inoculated control group for comparison. Both the test and the control samples contained the same polystyrene and inorganic salts medium. Hence, overlapping of peaks across these multiple salts and polystyrene substrate would be possible.

According to the spectrum at day 0 , the absorption peaks at $3400 \mathrm{~cm}^{-1}, 1044 \mathrm{~cm}^{-1}, 994 \mathrm{~cm}^{-1}, 926 \mathrm{~cm}^{-1}$, and $856 \mathrm{~cm}^{-1}$ have appeared with initial inoculation of ZM1 and TM1 (Figs. 7(a) and 7(b)). By comparing the spectral data onto Spectral Database for Organic Compounds (SDBS), these peaks were regarded as signal of glycerol, the remaining solution from ZM1 and TM1 bacterial stock (glycerol was used to preserve bacterial strain).

At the initial stage, the medium of inoculated groups share the same constituent of the control group, except glycerol from the bacterial stock solution. After 6 days of the experiment, weakening on multiple peaks occurs to the inoculated test groups compared to the control group. The reduced peaks seen in the range of $3000 \mathrm{~cm}^{-1}$ to $3100 \mathrm{~cm}^{-1}$ (Fig. 7) represent organic molecule functional groups including the band attributed to the aromatic $\mathrm{C}-\mathrm{H}$ stretch. Another absorption peak change in the test group occurred in the range of $2800 \mathrm{~cm}^{-1}$ to $3000 \mathrm{~cm}^{-1}$, which is assigned to the aliphatic $\mathrm{C}-\mathrm{H}$ stretch. Additionally, changes seen occurring at $1654 \mathrm{~cm}^{-1}, 1494 \mathrm{~cm}^{-1}$, and $1453 \mathrm{~cm}^{-1}$ are attributed to aromatic $\mathrm{C}=\mathrm{C}$ stretch. And a C-H in-plane bending peak change occurred at $1109 \mathrm{~cm}^{-1}$ in the test group. Aromatic rings and aliphatic $\mathrm{C}-\mathrm{H}$-rich backbones are two of the typical molecular features of polystyrene. 
Compared with the spectra of the un-inoculated control, the FTIR peak reductions seen in the carbon chain functional groups indicates that ZM1 and TM1 have the ability to degrade carbon in Polystyrene. For those polymers with long hydrocarbon backbones, it is possible that enzymes produced by TM1 and ZM1 are degrading polystyrene from the side chains of the molecular backbone itself (Fig. 8).

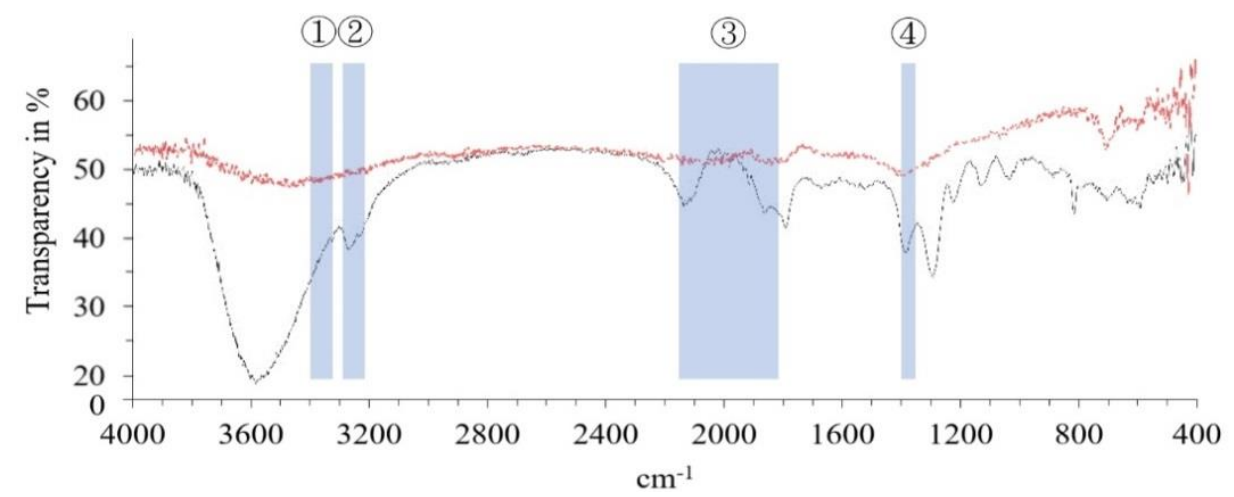

(a) Spectra with inoculation of strains ZM1 (day 0: black; day 6: red)

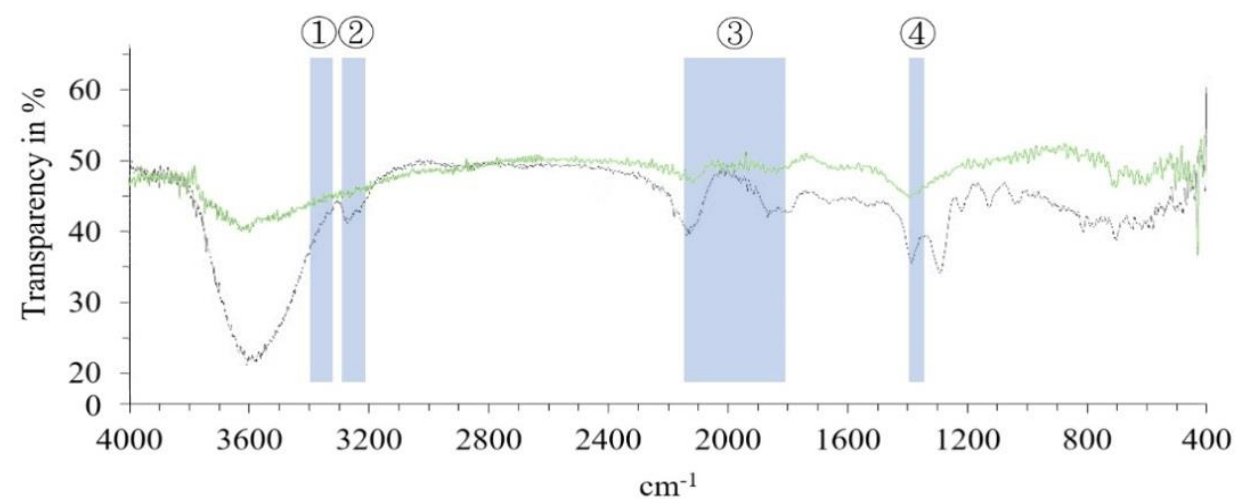

(b) Spectra with inoculation of strains TM1 (day 0: black; day 6: green)

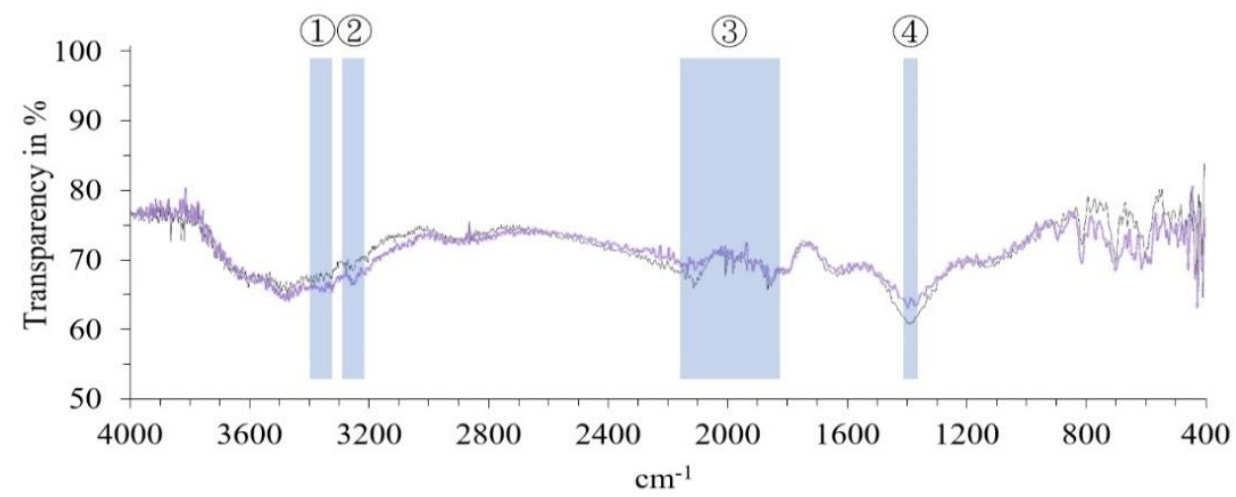

(c) Spectra of un-inoculated control (day 0: black; day 6: violet)

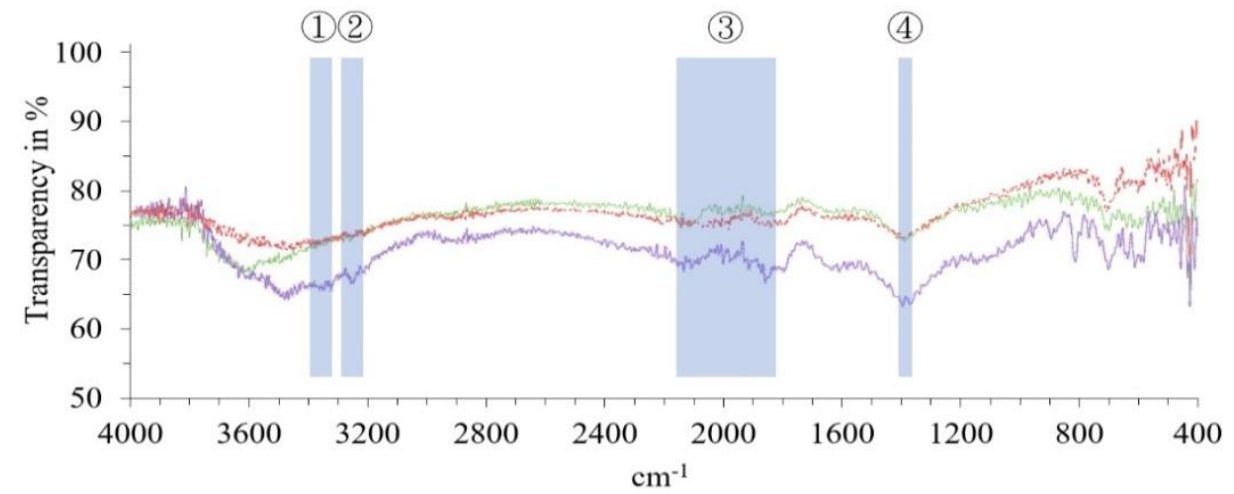

(d) Spectra of all three groups at day 6 (ZM1: red; TM1: green; control: violet)

Fig. 7 FTIR spectrum within the frequency range of $4000-400 \mathrm{~cm}^{-1}$; (1) refers to aromatic $\mathrm{C}-\mathrm{H}$ stretch; (2) refers to aliphatic $\mathrm{C}-\mathrm{H}$ stretch; (3) refers to aromatic $\mathrm{C}=\mathrm{C}$ stretch; (4) refers to $\mathrm{C}-\mathrm{H}$ in-plane bending 
The alteration or replacement of these side groups could create a new functional group, which would be feasible for other enzymes to break down along the main hydrocarbon chain. For example, research indicates that benzene 1,2-dioxygenase can degrade benzene (an aromatic derivative).

Considering that the aromatic rings of PS were also degraded in our study, TM1 and ZM1 may be shown to share a similar mechanism with benzene 1,2-dioxygenase degrading PS, by altering the benzene ring side chain.

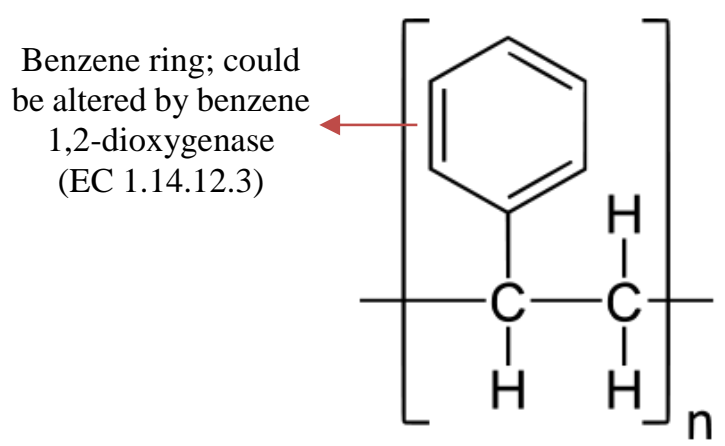

Fig. 8 molecular structure of polystyrene (PS), with a benzene side chain and $\mathrm{C}-\mathrm{H}$ rich backbone

\section{Conclusions}

To exclude the possibility of bacteria death causing the turbidity decrease, CFU tests were conducted and these showed no morbidity in the bacteria. With bacterial colony stable and turbidity decreasing, the inference is that the decrease in PS-medium turbidity was the result of digestion of PS emulsion. Turbidity assay as a means of verifying plastic biodegradation, as well as a means to quantify the efficiency of plastic decomposition, has been cited in other studies [11, 14-15].

Through FTIR analysis, multiple carbon bonding changes were revealed. These changes at the aromatic $\mathrm{C}-\mathrm{H}$ stretch; the aliphatic $\mathrm{C}-\mathrm{H}$ stretch; the aromatic $\mathrm{C}=\mathrm{C}$ stretch and the $\mathrm{C}-\mathrm{H}$ in-plane bending offer further evidence to suggest that it is the digestive processes of the bacteria strains TM1 and ZM1 in the PS-medium which were responsible for the alternations of the absorption peaks levels related to organic function seen [16-17].

Other research has discovered a potential manner for enzymatic degradation of polystyrene, utilizing hydroquinone peroxidase produced by Azotobacter beijerinckii HM121 [18]. Hydroquinone peroxidase is an enzyme which can oxidatively catalyze phenols such as hydroquinone, a derivative of benzene rings [19]. As a polymer molecular structure, it is notable that polystyrene is a rich in benzene rings as well [20]. Other studies [21-22] have mentioned the degradation ability of certain beetle larvae including the Tenebrio molitor, however the identification of the specific microbes and their enzymatic degradation mechanisms were not yet be described in detail.

In conclusion, our laboratory utilized the entire range of visible light wavelengths to scan turbidity via absorption and transparency modes to explore the optimal condition for future experiments. Through FTIR analysis Our laboratory revealed structural changes of the polystyrene at the sites above as the TM1 and ZM1 incubations which indicate biodegradation. Further studies offers researchers the chances to isolate, identify and potentially clone the specific intestinal enzymes that enable bacterial strains such as TM1 and ZM1 to break down polystyrene and expanded genetic engineering targets for this biodegrading enzymes.

\section{Conflicts of Interest}

The authors declare no conflict of interest. 


\section{References}

[1] S. S. Yang, A. M. Brandon, D. F. Xing, J. Yang, J. W. Pang, C. S. Criddle, et al., "Progresses in Polystyrene Biodegradation and Prospects for Solutions to Plastic Waste Pollution," IOP Conference Series: Earth and Environmental Science, vol. 150, pp. 1-10, May 2018.

[2] R. E. Schnurr, V. Alboiu, M. Chaudhary, R. A. Corbett, M. E. Quanz, K. Sankar, et al., "Reducing Marine Pollution from Single-Use Alastics (SUPs): A Review,” Marine Pollution Bulletin, vol. 137, pp. 157-171, December 2018.

[3] L. Yin, H. Liu, H. Cui, B. Chen, L. Li, and F. Wu, "Impacts of Polystyrene Microplastics on the Behavior and Metabolism in a Marine Demersal Teleost, Black Rockfish (Sebastes Schlegliei),” Journal of Hazardous Materials, vol. 380, pp. 120861 , December 2019.

[4] M. Revel, A. Châtel, and C. Mouneyrac, "Micro (nano) Plastics: A Threat to Human Health?” Current Opinion in Environmental Science \& Health, vol. 1, pp. 17-23, February 2018.

[5] Z. L. Tang, T. A. Kuo, and H. H. Liu, "The Study of the Microbes Degraded Polystyrene," Advanced Technology Innovation, vol. 2, pp. 13-17, 2017.

[6] B. Stuart, "FTIR of Biomolecules," Reviews in Cell Biology and Molecular Medicine, https://onlinelibrary.wiley.com/doi/abs/10.1002/3527600906.mcb.200300059, 2006.

[7] S. Bonhomme, A. Cuer, A. Delort, J. Lemaire, M. Sancelme, and G. Scott, "Environmental Biodegradation of Polyethylene," Polymer Degradation and Stability, vol. 81, no. 3, pp. 441-452, 2003.

[8] G. Chen, S. Liu, S. Chen, and Z. Qi, "FTIR Spectra, Thermal Properties, and Dispersibility of a Polystyrene/Montmorillonite Nanocomposite,” Macromolecular Chemistry and Physics, vol. 202, no. 7, pp. 1189-1193, April 2001.

[9] D. Olmos, E. Martin, and J. Gonzalez-Benito, "New Molecular-Scale Information on Polystyrene Dynamics in PS and PS-BaTiO 3 Composites from FTIR Spectroscopy," Physical Chemistry Chemical Physics, vol. 16, no. 44, pp. 24339-24349, 2014.

[10] H. Kundungal, M. Gangarapu, S. Sarangapani, A. Patchaiyappan, and S. P. Devipriya, "Efficient Biodegradation of Polyethylene (HDPE) Waste by the Plastic-Eating Lesser Waxworm (Achroia Grisella)," Environmental Science and Pollution Research, vol. 26, no. 18, pp. 18509-18519, May 2019.

[11] Yi-Feng Hsieh, "Degradation of Polyethylene Succinate by Thermophilic Microbispora sp. BC35T-4,” Master's dissertation, Department of Life Science, Fu-Jen University, 2008.

[12] H. K. Chan, B. Ongpipattanakul, and J. Au. Yeung, "Aggregation of rhDNase Occurred During the Compression of KBr Pellets Used for FTIR Spectroscopy,” Pharmaceutical Research, vol. 13, no. 2, pp. 238-242, February 1996.

[13] H. Auta, C. Emenike, and S. Fauziah, "Screening of Bacillus Strains Isolated from Mangrove Ecosystems in Peninsular Malaysia for Microplastic Degradation," Environmental Pollution, vol. 231, pp. 1552-1559, December 2017.

[14] J. Augusta, R. J. Müller, and H. Widdecke, “A Rapid Evaluation Plate-Test for the Biodegradability of Plastics," Applied Microbiology and Biotechnology, vol. 39, no. 4-5, pp. 673-678, July 1993.

[15] M. Timmins, D. Gilmore, N. Lotti, M. Scandola, R. Fuller, and R. Lenz, "A Spectrophotometric Method for Detection of Enzymatic Degradation of Thin Polymer Films,” Journal of Environmental Polymer Degradation, vol. 5, no. 1, pp. 1-15, 1997.

[16] M. Sobkowiak and P. Painter, "Determination of the Aliphatic and Aromatic CH Contents of Coals by FT-ir: Studies of Coal Extracts,” Fuel, vol. 71, no. 10, pp. 1105-1125, October 1992.

[17] A. N. A. El. Hendawy, "Variation in the FTIR Spectra of a Biomass under Impregnation, Carbonization and Oxidation Conditions," Journal of Analytical and Applied Pyrolysis, vol. 75, no. 2, pp. 159-166, March 2006.

[18] K. Nakamiya, G. Sakasita, T. Ooi, and S. Kinoshita, "Enzymatic Degradation of Polystyrene by Hydroquinone Peroxidase of Azotobacter Beijerinckii HM121," Journal of Fermentation and Bioengineering, vol. 84, no. 5, pp. 480-482, 1997.

[19] R. S. Yao, M. Sun, C. L. Wang, and S. S. Deng, "Degradation of Phenolic Compounds with Hydrogen Peroxide Catalyzed by Enzyme from Serratia Marcescens AB 90027," Water research, vol. 40, no. 16, pp. 3091-3098, September 2006.

[20] M. D. Faber, "Microbial Degradation of Recalcitrant Compounds and Synthetic Aromatic Polymers," Enzyme and Microbial Technology, vol. 1, no. 4, pp. 226-232, October 1979.

[21] B. Y. Peng, Y. Su, Z. Chen, J. Chen, X. Zhou, M. E. Benbow, et al., "Biodegradation of Polystyrene by Dark (Tenebrio Obscurus) and Yellow (Tenebrio Molitor) Mealworms (Coleoptera: Tenebrionidae)," Environmental Science \& Technology, vol. 53, no. 9, pp. 5256-5265, April 2019.

[22] Y. Yang, J. Wang, and M. Xia, "Biodegradation and Mineralization of Polystyrene by Plastic-Eating Superworms Zophobas Atratus," Science of the Total Environment, vol. 708, p. 135233, March 2020.

Copyright $(\mathrm{C}$ by the authors. Licensee TAETI, Taiwan. This article is an open access article distributed under the terms and conditions of the Creative Commons Attribution (CC BY-NC) license (https://creativecommons.org/licenses/by-nc/4.0/). 